\title{
14 \\ F.J. Clemens and Some Aspects of Neo-Scholasticism in the Education of F. Brentano
}

\author{
David Torrijos-Castrillejo
}

\begin{abstract}
Among the few publications which consider the Scholastic roots of Brentano's thinking, an article by Dieter Münch stands out. In it, he claims that the Aristotelian studies of Brentano and his whole philosophical project are inspired by the German Neo-Scholastic movement. Münch presents the Neo-Scholastic tendency as an ultra-conservative and reactionary program against modernity. Now, such a description makes almost inexplicable the fact that Brentano, who was educated in this context, could have developed a wholly personal and independent philosophy. To approach this framework objectively, I propose to pay attention to F.J. Clemens, a Neo-Scholastic who influenced Brentano's way of thinking. It is true that Clemens puts faith above reason and affirms that any conclusion obtained through the power of reason must be discarded when it contradicts faith. However, this is not an ultramontane innovation but simply the traditional theological point of view. He also argues that St. Thomas should be followed as a guide to orient oneself in the process of rational inquiry. Nevertheless, he doesn't want to understand philosophy as a repetition of Aquinas' claims; on the contrary, he rather proposes an intelligent use of tradition, which allows us to delve deeper into philosophical truth. For this very reason, he does not discard modernity or condemn it altogether. This perspective is also reflected in the early writings of Brentano, where he showed discontinuity from the Neo-Scholastic school of thought.
\end{abstract}

Keywords Franz Josef Clemens · Franz Brentano $\cdot$ Neo-Scholasticism • Ultramontanism $\cdot$ Catholicism

D. Torrijos-Castrillejo $(\square)$

University of San Dámaso, Madrid, Spain

D. Fisette et al. (eds.), Franz Brentano's Philosophy After One Hundred Years,

Primary Sources in Phenomenology, https://doi.org/10.1007/978-3-030-48563-4_14 


\section{References}

Albertazzi, Liliana. 2006. Immanent Realism: An Introduction to Brentano. Dordrecht: Springer.

Barnes, Jonathan. 2015. Brentano's Aristotle. In Method and Metaphysics. Essays in Ancient Philosophy I, ed. Maddalena Bonelli, 70-76. Oxford: Clarendon.

Baumgartner, Elisabeth, Wilhelm Baumgartner, and Klaus Hedwig. 2017. Franz Brentano Die Studienjahre. Brentano Studien 15: 17-142.

Bergmann, Hugo. 1946. Briefe Franz Brentanos an Hugo Bergmann. Philosophy and Phenomenological Research 7: 83-158.

Berti, Enrico. 2008. Aristotele nel Novecento. Roma: Laterza.

Binder, Thomas. 2017. "Vielleicht sagst Du, ich tüftele zu viel und Du würdest es nicht ganz mit Unrecht so sagen." Franz Brentanos Briefe an seine Tante Gunda von Savigny 1859-1861. Brentano Studien 15: 551-588.

Blum, Paul Richard. 1998. Franz Jakob Clemens e la lettura ultramontanistica di Bruno. In Brunus Redivivus. Momenti della fortuna di Giordano Bruno nel XIX secolo, ed. Eugenio Canone, 67-103. Pisa, Roma: Istituti Editoriali e Poligrafici Internazionali.

Brentano, Franz. 1968. Über die Zukunft der Philosophie nebst den Vorträgen Über die Gründe der Entmutigung auf philosophischen Gebiet, Über Schellings System sowie den 25 Habilitationsthesen, ed. Oskar Kraus. Hamburg: Meiner.

- 1867a. Geschichte der kirchlichen Wissenschaften. In Kirchengeschichte, ed. Johann Adam Möhler, vol. 2, 526-584. Regensburg: Manz.

- 1867b. Die Psychologie des Aristoteles, insbesondere seine Lehre vom NOY $\Sigma$ ПОIHTIKОГ: Nebst einer Beilage über das Wirken des Aristotelischen Gottes. Mainz: Franz Kirchheim.

1870. Thomas von Aquin. Rezension zu J. Delitzsch, Die Gotteslehre des Thomas von Aquin. Theologisches Literaturblatt 5: 459-463.

. 1895. Die vier Phasen der Philosophie und ihr augenblicklicher Stand. Stuttgart: Cotta. 1908. Thomas von Aquin. Neue Freie Presse (April 18).

Brock, Stephen L. 2015. The Philosophy of Saint Thomas Aquinas. A Sketch. Eugene: Cascade.

Burgess, Andrew J. 1974. Brentano as philosopher of religion. International Journal for Philosophy of Religion 5: 79-90.

Clemens, Franz Jakob. 1847. Giordano Bruno und Nicolaus von Cusa. Bonn: Wittmann.

-1856. De scholasticorum sententia philosophiam esse theologiae ancillam commentatio. Monasterii Guestphalorum: Aschendorff.

—. 1859a. Unser Standpunkt in der Philosophie. Der Katholik 39: 9-23. 129-154.

- 1859b. Die neueste Literatur über den heil. Thomas von Aquino. Der Katholik 39: 672-683. 803-823. 1025-1041. 1284-1299.

Kraus, Oskar. 1919. Franz Brentano: Zur Kenntnis seines Lebens und seiner Lehre: Mit Beiträgen von Carl Stumpf und Edmund Husserl. München: Beck.

L'Abbé, Petrus. 1674. Elogia Sacra: Theologica, et Philosophica. Regia. Eminentia. Illustria. Historica. Poetica. Miscellanea. Venetiis: Balleonium.

Liberatore, Matteo. 1857. Della conoscenza intellettuale, vol. 1. Roma: Civiltà Cattolica.

Münch, Dieter. 2004a. Franz Brentano und die katholische Aristoteles-Rezeption. In Phenomenology and Analysis: Essays in Central European Philosophy, ed. Arkadiusz Chrudzimski and Wolfgang Huemer, 159-198. Frankfurt: Ontos.

- 2004b. Franz Brentano et la réception catholique d'Aristote au XIX ${ }^{\mathrm{e}}$ siècle. In Aristote au XIX ${ }^{e}$ siècle, ed. Denis Thouard, 231-248. Villeneuve d'Ascq: Presses Universitaires du Septentrion.

Nettesheim, Josefine. 1962. Christoph Bernhard Schlüter und Franz Brentano. Zwei unbekannte Briefe Brentanos. Zeitschrift für Philosophische Forschung 16: 284-296.

Peitz, Detlef. 2006. Die Anfänge der Neuscholastik in Deutschland und Italien (1818-1870). Bonn: Nova \& Vetera. 
Piolanti, Antonio. 1988. Un Pioniere della filosofia cristiana della metà dell'Ottocento: Franz Jakob Clemens († 1862). Città del Vaticano: Libreria editrice Vaticana.

Russo, Antonio. 2003a. Franz Brentano e Heinrich Denifle: Un carteggio inedito. Studium 3: 333-356.

2003b. La Scuola cattolica di Franz Brentano: Heinrich Suso Denifle. Trieste: Edizioni Università di Trieste.

2004. Franz Brentano e Heinrich Denifle: alla scuola di Aristotele. In La filosofia come santità della ragione. Scritti in onore di Xavier Tilliette, ed. Antonio Russo and Jean-Louis Vieillard-Baron, 203-238. Trieste: Edizioni Università di Trieste.

- 2013. San Tommaso ed Aristotele nella formazione di Franz Brentano (1838-1917). Angelicum 90: 247-278.

. 2014. Franz Brentano. Heinrich Denifle: Un carteggio inedito. Roma: Studium.

2017. Il giovane Franz Brentano e Heinrich Denifle O.P. Brentano Studien 15: 373-413.

Schaefer, Richard. 2007a. Infallibility and Intentionality: Franz Brentano's Diagnosis of German Catholicism. Journal of the History of Ideas 68: 477-499.

- 2007b. Program for a New Catholic Wissenschaft: Devotional Activism and Catholic Modernity in the Nineteenth Century. Modern Intellectual History 4: 433-462.

Stöckl, Albert. 1870. Lehrbuch der Geschichte der Philosophie. Mainz: Kirchheim.

Tomasi, Pietro. 2007. The Unpublished "History of Philosophy" (1866-1867) by Franz Brentano. Axiomathes 17: 99-108.

. 2009. Una nuova lettura dell'Aristotele di Franz Brentano alla luce di alcuni inediti. Trento: Uni Service.

Torrijos-Castrillejo, David. 2016. Franz Brentano y Tomás de Aquino. Espíritu 65: 525-557.

— 2017. Propuestas de Franz Brentano para una correcta interpretación de Aristóteles. Pensamiento 75: 21-44.

Walter, Peter. 1988. Die neuscholastische Philosophie im deutschsprachigen Raum. Franz Jakob Clemens. In Christliche Philosophie im katholischen Denken des 19. und 20. Jahrhunderts, ed. Emerich Coreth, Walter M. Neidl, and Georg Pfligersdorffer, vol. 2, 134-139. Graz: Styria. 